\title{
Webquest como recurso tecnológico didático para capacitação de colaboradores em instituição hospitalar
}

Lívia Sanches Silva ${ }^{1}$, Dagmar Willamowius Vituri², Raquel Gvozd ${ }^{3}$, Renata Pedrão Motomatsu4, Maria do Carmo Fernandez Haddad ${ }^{5}$, Vivian El Reda Feijó 6

\section{RESUMO}

Neste estudo objetivou-se avaliar a utilização da Webquest como recurso tecnológico didático para capacitação quanto às Metas Internacionais de Segurança do Paciente em uma instituição hospitalar. Estudo quantitativo, transversal, realizado em hospital público de ensino, no Sul do Brasil. De 895 colaboradores, participaram da pesquisa 679 (76\%), vinculados à diretoria de enfermagem, sendo enfermeiros, técnicos em enfermagem, auxiliares de enfermagem, técnicos administrativos e auxiliares operacionais zeladores que foram capacitados quanto as metas de segurança. Foram avaliados 11 itens em cada meta aplicada, dentre eles apenas estrutura física, quantidade de textos, tempo destinado à capacitação, cores e design, aplicação na prática e objetividade dos textos foram referidos como regular ou ruim por um a $3 \%$ dos colaboradores. 0 recurso foi considerado satisfatório como estratégia para educação permanente, possibilitando a identificação de fragilidades do processo, passíveis de melhoria, para aprimoramento da metodologia Webquest em instituição hospitalar.

Descritores: Segurança do Paciente; Educação Continuada; Informática em Enfermagem.

\footnotetext{
${ }^{1}$ Enfermeira. Londrina, PR, Brasil. E-mail: lih_uel@hotmail.com.

${ }^{2}$ Enfermeira, Doutora em Enfermagem Fundamental. Enfermeira do Hospital Universitário da Universidade Estadual de Londrina. Londrina, PR, Brasil. E-mail: dagvituri@gmail.com.

${ }^{3}$ Enfermeira, Doutora em Enfermagem. Docente do Departamento de Enfermagem da Universidade Estadual de Londrina. Londrina, PR, Brasil. Email: raquelgvozd@yahoo.com.br.

${ }^{4}$ Enfermeira. Enfermeira do Hospital Universitário da Universidade Estadual de Londrina. Londrina, PR, Brasil. E-mail: renatapedrao@bol.com.br.

${ }^{5}$ Enfermeira, Doutora em Enfermagem Fundamental. Professora Associada do Departamento de Enfermagem da Universidade Estadual de Londrina. Londrina, PR, Brasil. E-mail: carmohaddad@gmail.com.

${ }^{6}$ Enfermeira, Mestre em Saúde Coletiva. Enfermeira do Hospital Universitário da Universidade Estadual de Londrina. Londrina, PR, Brasil. E-mail: feijovivif@gmail.com.
}

Artigo recebido: 15/06/2017. Artigo aprovado: 28/03/2018. Artigo publicado: 31/12/2018.

\section{Como citar esse artigo:}

Silva LS, Vituri DW, Gvozd R, Motomatsu RP, Haddad MCF, Feijó VR. Webquest como recurso tecnológico didático para capacitação de colaboradores em instituição hospitalar. Rev. Eletr. Enf. [Internet]. 2018 [acesso em: ];20:v20a43. Disponível em: https://doi.org/10.5216/ree.v20.47423. 


\section{INTRODUÇÃO}

Tendo em vista que os recursos humanos da instituição são responsáveis por planejar, desenvolver e executar todo o processo produtivo; dependem dos mesmos os produtos gerados e sua qualidade. Portanto, a capacitação dos colaboradores torna-se imprescindível para que o trabalho seja realizado conforme as necessidades da organização, com eficiência e eficácia.

Com o advindo da globalização e avanços tecnológicos, faz-se necessário novas estratégias de ensino que motivem e instiguem o desejo de buscar o conhecimento de forma inovadora. Nesta perspectiva, o recurso tecnológico Webquest, como estratégia de ensino, surgiu em 1995 e foi proposta por professores norte-americanos. Desde então tem sido aprimorada e utilizada para fins educativos em instituições de ensino, com o objetivo de formar profissionais ativos, que participem da busca pelo conhecimento, críticos e reflexivos ${ }^{(1)}$.

Webquest pode ser definida como investigação orientada, em que a maioria das informações, ou todas, são provenientes da internet ${ }^{(2)}$. $O$ indivíduo sente-se desafiado e se envolve no processo de aprendizado, a partir de um problema apresentado. A internet como ambiente de aprendizagem potencializa que $\mathrm{o}$ indivíduo transforme informação em conhecimento. Este recurso abrange o uso do senso crítico, síntese, análise, resolução de problemas e criatividade e, sendo assim, a qualidade do conteúdo é fundamental ${ }^{(3-4)}$.

A organização da Webquest é constituída por: introdução (contextualização e problematização do tema de forma clara e objetiva); objetivos (descrição do aprendizado esperado para o aluno); processo (tarefas que devem ser concluídas para o alcance dos objetivos; com a inserção de vídeos, sites e outras referências para consulta); avaliação (como será avaliada a aprendizagem do estudante); conclusão (estimular reflexão e síntese do conteúdo abordado) e créditos (apresentação das referências utilizadas para construção da Webquest) ${ }^{(1,3-4)}$.

Este recurso tecnológico pode ser classificado como curto, quando visa a integração do conhecimento, que deve ser explorado em uma a três aulas; ou longo, que se destina ao aprofundamento do conteúdo e refinamento do conhecimento, com duração de uma semana a um mês ${ }^{(4-5)}$.

No ambiente acadêmico é amplamente utilizado por professores e, na literatura são encontrados estudos que demonstram a utilização desta metodologia com alunos do ensino médio, graduação de enfermagem, na área da geografia e medicina ${ }^{(3-6)}$. No âmbito dos serviços de saúde o uso desta metodologia é inovador, dadas as peculiaridades do ambiente hospitalar e das especificidades da metodologia em si, que pressupõe o aprendizado ativo, por meio do uso de recursos da internet.

Tendo em vista a adequação da instituição para cumprimento dos requisitos estabelecidos no Brasil pelo Programa Nacional de Segurança do Paciente, Portaria GM/MS no 529/2013 que, dentre outras coisas, preconiza a qualificação do cuidado em todos os estabelecimentos de saúde do território nacional, por meio dos protocolos básicos de segurança do paciente ${ }^{(7)}$, estabeleceu-se investimentos maciços em capacitação dos colaboradores quanto aos mesmos.

Na prática assistencial é difícil retirar os colaboradores das unidades de trabalho durante o plantão, para sua capacitação; além de ser baixa a adesão dos mesmos às capacitações disponibilizadas fora do horário de trabalho. Assim, tendo em vista a necessidade de capacitar os profissionais quanto às Seis Metas Internacionais de Segurança do Paciente, com base nos novos protocolos institucionais, a Webquest foi utilizada como estratégia para envolver os colaboradores. 
Identificou-se na literatura poucos estudos referentes ao assunto e a maioria das publicações eram voltadas à academia $^{(3-6,8)}$. Tendo em vista a atual influência da tecnologia em diferentes áreas, sugere-se a utilização da Webquest para além de instituições de ensino.

Diante do exposto, o objetivo do estudo foi avaliar a utilização da Webquest como recurso tecnológico didático para capacitação quanto às Metas Internacionais de Segurança do Paciente em uma instituição hospitalar.

\section{MÉTOdO}

Estudo quantitativo, transversal, realizado em um hospital público de ensino do Sul do Brasil, de nível terciário, com 300 leitos destinados exclusivamente aos clientes do Sistema Único de Saúde (SUS). A população de estudo são os profissionais que compõem a diretoria de enfermagem da instituição, dos quais, 120 são enfermeiros, 294 técnicos em enfermagem, 276 auxiliares de enfermagem, 50 técnicos administrativos e 155 auxiliares operacionais zeladores. Os dados são provenientes de um banco de dados secundário, pertencente a Assessoria de Controle de Qualidade da Assistência de Enfermagem.

Antes do início da Webquest, os colaboradores eram convidados a participar da pesquisa como voluntários, assinando o Termo de Consentimento Livre e Esclarecido (TCLE), onde constavam o título da pesquisa, justificativa, objetivos, riscos e benefícios; deixando claro a liberdade de recusa e garantia de sigilo.

Os dados utilizados no presente estudo dizem respeito às respostas do instrumento de Avaliação de Reação, aplicado aos profissionais ao final de cada sessão de capacitação pela Webquest, referentes às Metas Internacionais de Segurança do Paciente: identificar corretamente o paciente; melhorar a comunicação entre profissionais de saúde; melhorar a segurança na prescrição, uso e administração de medicamentos; prática de higiene das mãos em serviço de saúde; risco de quedas e prevenção de lesão por pressão.

A coleta dos dados ocorreu no período de julho de 2015 a agosto de 2016. Cada profissional preencheu o instrumento composto por 11 questões, com respostas em uma escala do tipo Likert de cinco pontos (ótimo, bom, regular, ruim, péssimo), destinada à avaliação do método e qualidade do conteúdo, a saber: conteúdo, quantidade de textos, clareza e objetividade dos textos, cores e design da apresentação, facilidade no manuseio, aprendizado obtido, possibilidade de aplicação na prática, suporte recebido pelo tutor, período de tempo destinado, local onde foi realizada a capacitação, satisfação geral com a experiência; além de um campo para avaliação descritiva, com a possibilidade de sugestões.

Os dados foram tabulados em planilhas eletrônicas do programa Excel 2010, e calculadas as frequências simples e percentuais de cada resposta.

O estudo foi aprovado pelo Comitê de Ética em Pesquisa da Universidade Estadual de Londrina sob protocolo n으 258/2011.

\section{RESULTADOS}

No período da coleta de dados, o quadro de recursos humanos era composto por 294 técnicos em enfermagem, 276 auxiliares de enfermagem, 120 enfermeiros, 155 auxiliares operacionais zeladores e 50 técnicos administrativos, totalizando 895 profissionais, que constituíam a diretoria de enfermagem. 
A Tabela 1 apresenta a frequência e porcentagem de servidores capacitados pela Webquest, por meta e categoria profissional.

Tabela 1: Quantitativo de profissionais capacitados quanto às metas internacionais de segurança do paciente implantadas na instituição. Londrina, PR, Brasil, 2015/2016.

\begin{tabular}{|c|c|c|c|c|c|c|c|c|c|c|c|c|}
\hline \multirow{3}{*}{ Categoria Profissional } & \multicolumn{12}{|c|}{ Metas } \\
\hline & \multicolumn{2}{|c|}{1} & \multicolumn{2}{|c|}{2} & \multicolumn{2}{|c|}{3} & \multicolumn{2}{|c|}{4} & \multicolumn{2}{|c|}{5} & \multicolumn{2}{|c|}{6} \\
\hline & $\mathbf{n}$ & $\%$ & $\mathbf{n}$ & $\%$ & $\mathbf{n}$ & $\%$ & $\mathbf{n}$ & $\%$ & $\mathbf{n}$ & $\%$ & $\mathbf{n}$ & $\%$ \\
\hline Técnico de enfermagem & 248 & 84 & 226 & 77 & 209 & 71 & 218 & 74 & 253 & 86 & 271 & 92 \\
\hline Auxiliar de enfermagem & 223 & 81 & 205 & 74 & 171 & 62 & 181 & 66 & 176 & 64 & 199 & 72 \\
\hline Enfermeiro & 89 & 74 & 98 & 82 & 96 & 80 & 84 & 70 & 83 & 69 & 100 & 83 \\
\hline Auxiliar operacional & 54 & 35 & 70 & 45 & 27 & 17 & 16 & 10 & 21 & 14 & 11 & 7 \\
\hline Técnico administrativo & 39 & 78 & 38 & 76 & 37 & 74 & 26 & 52 & 15 & 30 & 0 & 0 \\
\hline Total & 679 & 76 & 656 & 73 & 585 & 65 & 560 & 63 & 561 & 63 & 581 & 65 \\
\hline
\end{tabular}

Os colaboradores que não conseguiram realizar a Webquest no período estabelecido, seja porque estavam em férias e/ou licenças, tiveram a oportunidade de participar da repescagem, totalizando 138 funcionários.

A Tabela 2 demonstra os resultados observados quanto à avaliação realizada referente ao tempo destinado a capacitação e o local de sua realização.

Tabela 2: Distribuição da avaliação dos colaboradores com relação às Webquest referentes às seis metas internacionais de segurança do paciente aplicadas quanto à estrutura. Londrina, PR, Brasil, 2015/2016.

\begin{tabular}{ccccccccccccc}
\hline \multirow{2}{*}{ Itens de Avaliação } & \multicolumn{2}{c}{ Ótimo } & \multicolumn{2}{c}{ Bom } & \multicolumn{2}{c}{ Regular } & \multicolumn{2}{c}{ Ruim } & \multicolumn{2}{c}{ Péssimo } & \multicolumn{2}{c}{ Total } \\
\cline { 2 - 14 } & $\mathbf{n}$ & $\mathbf{\%}$ & $\mathbf{n}$ & $\mathbf{\%}$ & $\mathbf{n}$ & $\mathbf{\%}$ & $\mathbf{n}$ & $\mathbf{\%}$ & $\mathbf{n}$ & $\mathbf{\%}$ & $\mathbf{N}$ & $\mathbf{\%}$ \\
\hline Tempo destinado & 1461 & 54 & 1113 & 41 & 108 & 04 & 18 & 01 & 05 & 00 & 2705 & 100 \\
Local de realização & 1423 & 53 & 1081 & 40 & 146 & 05 & 38 & 01 & 18 & 01 & 2706 & 100 \\
\hline
\end{tabular}

Dos 11 itens avaliados em cada uma das seis metas aplicadas pela Webquest, mais de $90 \%$ foram considerados como ótimo ou bom. Quanto aos itens relacionados à apresentação do conteúdo abordado, conforme expõe a Tabela 3, destaca-se como ótimo: cores e design da apresentação (60\%) e o conteúdo da capacitação (71\%); como regular: clareza e objetividade dos textos (3\%) e ruim: quantidade de textos (1\%), conforme demonstra a Tabela 3.

Tabela 3: Distribuição da avaliação dos colaboradores com relação às webquest referentes às seis metas internacionais de segurança do paciente aplicadas quanto ao processo. Londrina, PR, Brasil, 2015/2016.

\begin{tabular}{|c|c|c|c|c|c|c|c|c|c|c|c|c|}
\hline \multirow{2}{*}{ Itens de Avaliação } & \multicolumn{2}{|c|}{ Ótimo } & \multicolumn{2}{|c|}{ Bom } & \multicolumn{2}{|c|}{ Regular } & \multicolumn{2}{|c|}{ Ruim } & \multicolumn{2}{|c|}{ Péssimo } & \multicolumn{2}{|c|}{ Total } \\
\hline & $\mathbf{n}$ & $\%$ & $\mathbf{n}$ & $\%$ & $n$ & $\%$ & $\mathbf{n}$ & $\%$ & $\mathbf{n}$ & $\%$ & $\mathbf{N}$ & $\%$ \\
\hline Conteúdo & 1888 & 71 & 757 & 28 & 16 & 01 & 01 & 00 & 00 & 00 & 2662 & 100 \\
\hline Clareza e Objetividade & 1718 & 64 & 901 & 33 & 78 & 03 & 05 & 00 & 00 & 00 & 2702 & 100 \\
\hline Quantidade de textos & 1462 & 54 & 1160 & 42 & 89 & 03 & 15 & 01 & 02 & 00 & 2728 & 100 \\
\hline Cores e design & 1616 & 60 & 986 & 37 & 91 & 03 & 09 & 00 & 01 & 00 & 2703 & 100 \\
\hline Facilidade no manuseio & 1696 & 63 & 935 & 35 & 62 & 02 & 07 & 00 & 01 & 00 & 2701 & 100 \\
\hline
\end{tabular}

Conforme apresenta a Tabela 4, dos itens relacionados à satisfação do colaborador com relação à capacitação, foram classificados como ótimo o suporte recebido pelo tutor (79\%) e a satisfação geral com a experiência (65\%). A aplicação na prática foi considerada como regular por 69 colaboradores (3\%). 
Tabela 4: Distribuição da avaliação dos colaboradores com relação às webquest referentes às seis metas internacionais de segurança do paciente aplicadas quanto ao resultado. Londrina, PR, Brasil, 2015/2016.

\begin{tabular}{|c|c|c|c|c|c|c|c|c|c|c|c|c|}
\hline \multirow{2}{*}{ Itens de Avaliação } & \multicolumn{2}{|c|}{ Ótimo } & \multicolumn{2}{|c|}{ Bom } & \multicolumn{2}{|c|}{ Regular } & \multicolumn{2}{|c|}{ Ruim } & \multicolumn{2}{|c|}{ Péssimo } & \multicolumn{2}{|c|}{ Total } \\
\hline & $n$ & $\%$ & n & $\%$ & $\mathbf{n}$ & $\%$ & $\mathbf{n}$ & $\%$ & $\mathbf{n}$ & $\%$ & $\mathbf{N}$ & $\%$ \\
\hline Aprendizado & 1772 & 66 & 897 & 33 & 30 & 01 & 03 & 00 & 03 & 00 & 2705 & 100 \\
\hline Aplicação prática & 1741 & 65 & 875 & 32 & 69 & 03 & 09 & 00 & 00 & 00 & 2694 & 100 \\
\hline Suporte recebido pelo tutor & 2125 & 79 & 545 & 20 & 17 & 01 & 06 & 00 & 02 & 00 & 2695 & 100 \\
\hline Satisfação com experiência & 1754 & 65 & 912 & 34 & 39 & 01 & 03 & 00 & 02 & 00 & 2710 & 100 \\
\hline
\end{tabular}

Do campo descritivo disponível para sugestões foram analisados 482 comentários, estratificados em estrutura, processo e resultado.

Com relação à estrutura, ao local e tempo destinados à capacitação, envolveram 7\% (35) dos comentários, dos quais 66\% (25) foram críticas sobre a realização da capacitação no setor, devido ao local ser inadequado, com interrupções e ruídos. No entanto, $17 \%$ (06) referiram ser positiva a realização da capacitação no horário e unidade de trabalho.

No que se refere ao processo, $18 \%$ (86) foram sugestões diretamente ligadas à mudanças na prática profissional, 35\% (167) à metodologia, como questões confusas, textos longos e cansativos, necessidade de ajustes no tamanho da fonte e slides, e a sugestão de disponibilização do material por determinado período no sistema informatizado da instituição.

Quanto ao resultado, 42\% (202) emitiram comentários, sendo 5\% (10) críticas envolvendo questionamentos quanto à aplicação do conteúdo à prática, sendo que 95\% (192) destes comentários foram positivos, com relação a boa qualidade do conteúdo abordado, metodologia utilizada, importância de se manter as capacitações para reflexão da prática e atualização profissional.

\section{DISCUSSÃO}

Dos 11 itens avaliados pelos participantes do estudo, a maioria foi considerada entre ótimo ou bom, caracterizando a experiência vivenciada com a Webquest como estratégia de capacitação positiva por grande parte dos colaboradores. Porém, apesar das facilidades adquiridas com a capacitação do colaborador sem o retirar de seu ambiente de trabalho, o estudo demonstrou algumas fragilidades que devem ser avaliadas para o aprimoramento da experiência com este novo modelo de educação permanente.

Atualmente todas as áreas sofrem influência da tecnologia, porém a área da saúde em especial necessita de profissionais que busquem aprimoramento constante de conhecimentos, favorecendo sua autonomia e minimização do risco de erros. A informática é uma ferramenta que otimiza o processo do cuidado, seu gerenciamento, ensino, pesquisa ${ }^{(9)}$, e possui influência na qualidade da assistência prestada. Neste sentido, a Webquest é um recurso que pode ser utilizado para educação em serviço em instituições hospitalares, como demonstra o presente estudo.

Frente a este cenário, a preocupação com a segurança do paciente durante seu período de internação tem se tornado cada vez mais frequente nas instituições hospitalares ${ }^{(10-11)}$. Portanto faz-se necessário uma equipe qualificada e devidamente orientada quanto aos riscos mais frequentes aos quais os pacientes encontram-se 
expostos na tentativa de reduzi-los, pois, os mesmos podem ocasionar danos irreparáveis à vida, podendo resultar em óbito(12).

A segurança do paciente visa redução do risco de danos desnecessários relacionados à assistência à saúde, denominados incidentes: circunstâncias que podem resultar em complicações dispensáveis ao cliente, decorrentes da assistência à saúde fornecida. Podem ser classificados em incidentes sem dano, ou com dano; quando causa dano físico, social ou psicológico ao indivíduo, também denominado evento adverso(13).

Estes ocasionam prejuízos à sociedade, paciente e familiar, com o aumento da morbimortalidade, a depender de sua gravidade, resultando no prolongamento do período de internação, gastos com medicações, diária em unidade de internação ou unidade de terapia intensiva(12).

Tendo em vista o direito do cliente de receber uma assistência de enfermagem adequada, minimizando as chances de danos ao mesmo; é imprescindível, além da atualização profissional, o seu envolvimento com o movimento global pela segurança do paciente e sua corresponsabilização em relação à prestação de um cuidado seguro e, portanto, de qualidade ${ }^{(14-15)}$.

Neste contexto, a instituição estudada optou pela utilização da Webquest enquanto recurso tecnológico, como estratégia para capacitação dos colaboradores da Diretoria de Enfermagem quanto às seis metas internacionais de segurança do paciente.

Algumas das Webquests realizadas na pesquisa, em função das particularidades da meta abordada, como a de cirurgia segura e prevenção de lesão por pressão, com conteúdo extenso e mais complexo, receberam avaliações negativas em alguns aspectos.

As capacitações realizadas na unidade de trabalho do colaborador durante seu turno geram algumas preocupações com relação à interrupção da assistência aos clientes. Contudo, houve avaliações positivas em relação à capacitação neste ambiente, devido à dificuldade de se ausentar do setor ou comparecer em outro horário para a realização da mesma. É um desafio retirar o colaborador do ambiente de trabalho, devido às intensas e inúmeras atividades realizadas no setor, recursos humanos escassos, absenteísmo e readequações profissionais.

A realização da capacitação no local de trabalho proporciona a redução do tempo utilizado para o deslocamento até o local de treinamento, e o colaborador sente-se seguro por ser um ambiente familiar. Interfere na diminuição dos níveis de ansiedade facilitando o processo de aprendizagem ${ }^{(16)}$.

Apesar disso, é necessário avaliar a influência da capacitação realizada fora de seu ambiente de trabalho, a qual possibilita ao trabalhador a oportunidade de conhecer um novo espaço, outros funcionários, visualizar outros contextos e incorporar o conhecimento adquirido ao seu dia-a-dia ${ }^{(14)}$. Mas, para isso, o enfermeiro necessita perceber a capacitação como uma necessidade de aprendizado do funcionário e o motivar a participar.

Tendo em vista a importância da capacitação de todos os colaboradores quanto às metas internacionais de segurança do paciente, com intuito de possibilitar aos funcionários que no período da capacitação estavam ausentes por absenteísmo previsto ou não previsto, a instituição em estudo ofertou a oportunidade da realização da Webquest em outro momento (repescagem). 


\section{CONCLUSÃO}

A avaliação realizada pelos participantes da capacitação possibilitou uma visão ampla dos benefícios alcançados, como a qualificação de grande parte dos profissionais com relação ao conteúdo proposto. Demonstrou a satisfação dos mesmos quanto à utilização deste recurso tecnológico na instituição hospitalar como estratégia para a educação permanente em saúde e possibilitou a identificação de fragilidades passíveis de melhoria, para que a capacitação em seu turno e unidade de trabalho se torne uma metodologia eficaz.

Identificou-se que a capacitação no ambiente e horário de trabalho facilita a participação do colaborador, porém, faz-se necessário que o mesmo possua condições apropriadas para este tipo de atividade. É preciso o preparo de um ambiente confortável, livre de ruídos e que seja destinado especificamente para capacitações, associadas à outras estratégias para potencializar e motivar o colaborador.

Verificou-se com este estudo que a utilização da Webquest ainda está concentrada em instituições de ensino e pouco em estratégias de educação em saúde. Tendo em vista a atual influência da tecnologia em diferentes áreas, sugere-se a Webquest para além de instituições de ensino.

$\mathrm{Na}$ área da saúde especificamente, a qualidade da assistência prestada ao cliente depende diretamente da capacitação da equipe envolvida, sendo esta responsabilidade da instituição. Desta forma, o presente estudo demonstra a possibilidade de ampliar a utilização da estratégia Webquest para o uso na capacitação em instituições de saúde, como recurso estratégico para educação em serviço.

Remete-se o êxito na execução da referida estratégia na instituição em estudo, o fato de ser de ensino, o que pode ser um facilitador para a incorporação de novas tecnologias, recursos humanos e materiais disponíveis. Portanto, sugere- se a realização de estudos semelhantes em instituições com realidades diferentes da estudada, a fim de avaliar a viabilidade da utilização deste recurso tecnológico didático em instituições que não tenham cunho de ensino.

\section{REFERÊNCIAS}

1. Bottentuit Junior JB, Coutinho CP. Recomendações de qualidade para o processo de avaliação de webquest. Ciênc Cogn [Internet]. 2012 [acesso em: 15 out 2017];17(1):73-82. Disponível em:

http://www.cienciasecognicao.org/revista/index.php/cec/article/view/754/524.

2. Silva NM, Bottentuit Junior JB. Uma proposta de uso da metodologia webquest para o ensino e aprendizagem de literatura. Rev Renote [Internet]. 2014 [acesso em: 15 out 2017];12(1). Disponível em: http://seer.ufrgs.br/index.php/renote/article/view/50341. 3. Pereira MCA, Melo MRAC, Silva ASB, Évora YDM. Avaliação da webquest gerenciamento de recursos materiais em enfermagem por alunos do curso de graduação. Rev Latino-Am Enferm [Internet]. 2010 [acesso em: 15 out 2017];18(6):1107-14. Disponível em: http://dx.doi.org/10.1590/S0104-11692010000600010.

4. Maruxo HB, Prado C, Almeida DM, Tobase L, Grossi MG, Vaz DR. Webquest e história em quadrinhos na formação de recursos humanos em enfermagem. Rev Esc Enferm USP [Internet]. 2015 [acesso em: 20 out 2017]; 49(esp.2):68-74. Disponível em: http://dx.doi.org/10.1590/S0080-623420150000800010.

5. Archela RS, Pissinati MC. Webquest como metodologia em aulas práticas de cartografia. Portal Cartografia [Internet] 2008 [acesso em: 20 out 2017];1(1):59-74. Disponível em:

http://www.uel.br/revistas/uel/index.php/portalcartografia/article/view/1359.

6. Mondeja LDAM, Suárez LAD, Vázquez LA. Webquest: enfermidades infecciosas en situaciones de desastres naturales. RCIM [Internet]. 2015 [acesso em: 20 out 2017];7(1):31-9. Disponível em: http://scielo.sld.cu/pdf/rcim/v7n1/rcim04115.pdf.

7. Ministério da Saúde (BR). Portaria no. 529, de 10 de abril de 2013. Institui o Programa Nacional de Segurança do Paciente (PNSP) [Internet]. Diário Oficial da União. 2013. [acesso em: 15 out 2017]. 02 de abril de 2013. Disponível em:

http://bvsms.saude.gov.br/bvs/saudelegis/gm/2013/prt0529 0104 2013.html. 
8. Jahromi ZB, Mosalanejad L, Rezaee R. O efeito da busca na Web e da aprendizagem em equipe na auto-regulação dos alunos. J Adv Med Educ Prof [Internet]. 2016 [acesso em: 19 março 2018]; 4(2):80-87. Disponível em:

https://www.ncbi.nlm.nih.gov/pmc/articles/PMC4827760/.

9. Juliani CMCM, Silva MC, Bueno GH. Avanços da informática em enfermagem no Brasil: revisão integrativa. J Health Inform [Internet]. 2014 [acesso em: 15 out 2017];6(4):161-5. Disponível em: http://www.jhi-sbis.saude.ws/ojs-jhi/index.php/ihisbis/article/view/322/218.

10. Hinrichsen SL, Possa SL, Oliveira CLF, Ramos DM, Vilella TAS. Análise de modos e efeitos de falhas (FMEA) e metas internacionais de segurança do paciente: estudo piloto. RAS [Internet]. 2012 [acesso em: 15 out 2017];14(57):151-60.Disponível em: http://www.cqh.org.br/portal/pag/doc.php?p ndoc=515.

11. Franciscatto L, Bessow CK, Ruzczyk JVA, Oliveira MA, Kluck MM. Metas internacionais de segurança do paciente em hospital universitário. Rev HCPA [Internet]. 2011[acesso em: 10 out 2017];31(4):482-6. Disponível em:

http://seer.ufrgs.br/hcpa/article/view/21146/14967.

12. Nascimento NB, Travassos CMR. O erro médico e a violação às normas e prescrições em saúde: uma discussão teórica na área de segurança do paciente. Physis [Internet]. 2010 [acesso em:15 out 201];20(2):625-51. Disponível em:

http://dx.doi.org/10.1590/S0103-73312010000200016.

13. Novaretti MCZ, Santos EV, Quitério LM, Gallotti RMD. Sobrecarga de trabalho da enfermagem e incidentes e eventos adversos em pacientes internados em UTI. Rev Bras Enferm [Internet]. 2014 [acesso em: 15 out 2017];67(5):692-9. Disponível em: http://dx.doi.org/10.1590/0034-7167.2014670504.

14. Silva GM, Seiffert OMLB. Educação continuada em enfermagem: uma proposta metodológica. Rev Bras Enferm [Internet]. 2009 [acesso em: 20 out 2017];362-36. Disponível em: http://dx.doi.org/10.1590/\$0034-71672009000300005.

15. Vargas MAO, Luz AMH. Práticas seguras do/no cuidado de enfermagem no contexto hospitalar: é preciso pensar sobre isso e aquilo. Enferm Foco [Internet]. 2010 [acesso em: 20 out 2017];1(1):23-7. Disponível em:

http://revista.portalcofen.gov.br/index.php/enfermagem/article/viewFile/5/6.

16. Costa DB, Vannuchi MTO, Haddad MCFL, Cardoso MGP, Silva LG, Garcia SD. Custo da educação continuada para equipe de enfermagem de um hospital universitário público. Rev Eletr Enf [Internet]. 2012 [acesso em: 20 out 2017];14(2):257-66. Disponível em: http://dx.doi.org/10.5216/ree.v14i2.14540. 